\title{
Meta-Heuristic Ant Colony Algorithm for Multi-Tasking Assignment on Collaborative AUVs
}

\author{
Jian Jun $\mathrm{Li}^{1,3}$, Ru Bo Zhang ${ }^{1,2}$ and Yu Yang ${ }^{3}$ \\ ${ }^{1}$ College of Computer Science and Technology, Harbin Engineering \\ University, Harbin 150001, China \\ ${ }^{2}$ College of Electromechanical \& Information Engineering, Dalian \\ Nationalities University, Liaoning Dalian, 116600, China \\ ${ }^{3}$ School of Computer and Information Engineering, Harbin University of \\ Commerce, Harbin 150028, China) \\ Cn_lijj@126.com
}

\begin{abstract}
Multiple Unmanned Underwater Vehicles Is a typical combinatorial optimization problem, to achieve multiple AUV, coordinated, collaborative tasks to complete complex jobs subsea. Through analyzing the ant colony optimization algorithm, the paper proposed An Meta-heuristic ant colony optimization algorithm the Implementation to solve the multi AUVs to achieve the task allocation problem, and had simulation test based on the consolidated analyze the advantages of multiple unmanned underwater vehicle .results show that the ant colony optimization algorithms in solving multi-task allocation problem of multiple unmanned underwater vehicle showed a good performance.
\end{abstract}

Keywords; Ant Colony Optimization, Multi-AUVs, Task Assignment, Meta-Heuristic Algorithm

\section{Introduction}

Due to the complex subsea operating environment, many undersea target in the form of discrete distributed in different regions, a single AUV (Autonomous underwater vehicle, AUV) moving away from the target due to the angle, speed, travel distance and other factors limit, Difficult to accomplish alone complex underwater target search task. So take advantage of multi- AUV system (MultiAutonomous underwater vehicle system, AUVs) synergistic complex task allocation, Become one of the multiple AUVs key research direction. Zhu Da qi et al., proposed based on self-organizing map (SOM) neural network multi- AUV multi-objective allocation strategy [1]. Sophie, who proposed the division of the mechanism based on ant colony algorithm for non- human-machine cooperative multitasking assignment solved [2]. Chen Jing, who for parallel and distributed systems of the same type machine scheduling problem, an improved ant colony algorithm [3]. Italian scholar Dorigo proposed the basic model (Ant Colony Optimization, ACO) ant colony algorithm in 1992 It is a search for the most optimal path of probabilistic algorithms in the figure [4]. Ant colony algorithm uses a distributed parallel computer system, has strong robustness. Assignment ant colony behavior is one of the more significant features; ants can be divided in the breeding, foraging, nesting, such as different individuals, these individuals reasonable task allocation in the absence of any information to guide case. Features dynamic equilibrium exists in the scientific process to solve the task allocation for ant colony algorithm, Presents a meta-heuristic ant colony optimization algorithm to solve the problem of multi- tasking AUVs implementation method, the experimental results 
show that this method has good performance. So study the application of ant colony algorithm to solve multiple AUVs cooperative task assignment has a very important practical significance and promotional value.

\section{Ant Colony Optimization}

\subsection{Aco Meta-Heuristic Algorithm}

ACO algorithm is a meta-heuristic algorithm for discrete optimization problems, which uses a group of artificial ants to search for the key elements of the optimal solution. ACO algorithm design is collaboration, It does this by allocating computing resources on a group of simple artificial ant agent, agent collaboration between these artificial ants find the optimal solution through indirect implicit forms of communication.

ACO meta heuristic basic framework for interaction by the process consisting of three operationspp. Building Solutions, pheromone update, the background [5].

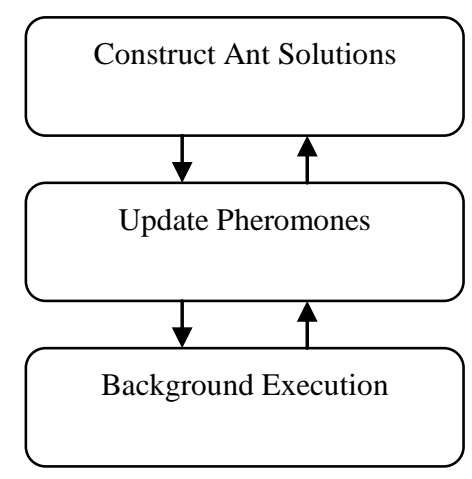

\section{Figure 1. The Basic Framework of ACO Meta Heuristic}

(1) Construct Ant Solutionspp. a group based on artificial ants pheromone and heuristic information, using a random selection of local decision-making the next move, Gradually establish a complete solution of the optimization problem, and solution of quality assessment constituted so much pheromone next procedure should be released.

(2) Update Pheromonespp. Is pheromone concentration process of change, including the pheromone release and evaporation process increases the release of a new artificial ant pheromone probability of selection of a point and an edge while pheromone evaporation usually a fixed percentage of the value gradually. Attenuation all sides of the pheromone, avoidance algorithm is not optimal solution towards premature convergence, so that the algorithm can search for solutions of more new areas.

(3) Background Executionpp. Macro operations perform a single ant cannot be completed, such as the implementation of a global gathering pheromone circumstances and local optimization process, etc., the whole algorithm optimization is performed by regulating the behavior of the background. ACO meta-heuristic algorithm has been applied to many different combinatorial optimization problems, including static and dynamic combinatorial optimization problems of combinatorial optimization problems. Tasking is a typical combinatorial optimization problem, so the use of ACO metaheuristic algorithm can effectively solve the task allocation problem. 


\subsection{ACO Mathematical Model}

Set $b_{i}(t)$ is the number of ants in the time $t i$ of the target, $\tau_{i j}(t)$ For $t$ time goal $(i, j)$ The amount of pheromone on. $n$ Is the number of target size, $m$ Ant quantity scale, Then $m=\sum_{i=1}^{n} b_{i}(t) ; \Gamma=\left\{\tau_{i j}(t) \mid c_{i}, c_{j} \subset C\right\}$ is the target set at time $t$ Target collection $C$ In twenty-two target connection $l_{i j}$ The residual amount of information collection. Initial time $\tau_{i j}(0)=$ const , The basic ant colony optimization algorithm by a directed graph $g=(C, L, \Gamma)$ Implementation [5].

ant $k(k=1,2, \cdots, m)$ Determine its direction of movement of the connection according to the amount of information on each strip, You can use the tabu list $\operatorname{lab}_{k}(k=1,2, \cdots, m)$ Record Ants $k$ Search through the target point, With the target collection $t a b u_{k}$ The evolution of dynamic adjustment. Ants can always calculate transition probabilities based on pheromone path information and inspiration $p_{i j}^{k}(t)$ [6].

$$
p_{i j}^{k}(t)=\left\{\begin{array}{l}
\frac{\left[\tau_{i j}(t)\right]^{\alpha} \square\left[\eta_{\mathrm{ik}}(t)\right]^{\beta}}{\sum_{\text {scallowed }_{k}}\left[\tau_{i s}(t)\right]^{\alpha} \square\left[\eta_{\mathrm{is}}(t)\right]^{\beta}}, j \in \text { allowed }_{k} \\
0, \quad \text { otherwise }
\end{array}\right.
$$

Equation (1.1) allowed $d_{k}=\left\{C-t a b u_{k}\right\}$ Under the ant $\mathrm{k}$ can choose a target ; $\alpha$ for information heuristic factor, the greater its value, the greater the tendency of ants choose other routes, the stronger collaboration between ants; $\beta$ is the desired heuristic factor .

$\eta_{i j}(t)$ for the heuristic function, its expression is as follows pp.

$$
\eta_{i j}(t)=\frac{1}{d_{i j}}
$$

$d_{i j}$ Represents the distance between two points adjacent.$d_{i j}$ The smaller, $\eta_{i j}(t)$ The larger, The larger the $p_{i j}^{k}(t)$, Ants select from the target $i$ to the target $j$ higher expectations.

Due to the presence of volatile pheromone and other factors, So the need for $t+n$ Time path $(i, j)$ The amount of information to be adjusted.

$$
\begin{gathered}
\tau_{i j}(t+n)=(1-\rho) \square_{\tau_{i j}}(t)+\square \tau_{i j}(t) \\
\square \tau_{i j}(t)=\sum_{k=1}^{m} \square \tau_{i j}^{k}(t)
\end{gathered}
$$

$\rho$ Is the pheromone evaporation rate, $1-\rho$ Is the residual factor pheromone ,$\rho \subset[0,1] ; \square_{i j}(t)$ Is this cycle pheromone increment, Initial time $\square_{\tau_{i j}}(0)=0, \quad \square \tau_{i j}^{k}(t)$ $k$ denotes the ant to stay in the path $(i, j)$ Information. 
Dorigo Depending on the pheromone update strategy, proposed three different basic ant colony algorithm model arepp.

Ant-Cycle model

$$
\square_{\tau_{i j}^{k}}(t)= \begin{cases}\frac{Q}{L_{K}} & \\ 0, & \text { otherwise }\end{cases}
$$

Ant-Quantity model

$$
\square_{\tau_{i j}^{k}}(t)=\left\{\begin{array}{l}
\frac{Q}{d_{i j}} \\
0, \quad \text { otherwise }
\end{array}\right.
$$

Ant-Density model

$$
\square_{\tau_{i j}^{k}}(t)=\left\{\begin{array}{l}
Q \\
0, \quad \text { otherwise }
\end{array}\right.
$$

$Q$ represents the elapsed between pheromone strength $(i, j) \quad k$ - ant $t$ and $t+1$ time . Of formula (1.5) is the overall use of the information and the formula (1.6) and (1.7) using the local information.

\section{Multi- Tasking AUVS}

\subsection{Generalized Assignment}

Generalized Assignment Problem, GAP, That is, a collection of tasks $\mathrm{i}(\mathrm{i} \in I)$ Agent assigned to a collection $\mathbf{j}(\mathbf{j} \in J)$ Execution is completed. Assigned goal is to find the cheapest price consuming task allocation scheme.

$$
\begin{gathered}
\min f(y)=\sum_{j=1}^{m} \sum_{i=1}^{n} d_{i j} y_{i j} \\
\text { s.t } \\
\sum_{i=1}^{n} r_{i j} y_{i j} \leq a j,(i=1, \ldots, m) \\
\sum_{j=1}^{m} y_{i j}=1,(i=1, \ldots, n) \\
y_{i j} \in\{0,1\},(i=1, \ldots, n ; j=1, \ldots, m)
\end{gathered}
$$

$d_{i j}$ is Each task $i$ Assigned to agent $j$ When the cost of consumption, $r_{i j}$ is Each task $i$ Assigned to agent $j$ The energy consumption, the objective function is the task allocation scheme costs that consume a minimum.

When the task set $i$ is assigned to agent $j, \quad y_{i j}$ a value of 1 , else $y_{i j}$ the value of 0. Constraint equation (2.2) indicates that agent with limited energy. Equation (2.3) (2.4) indicates that a task can be assigned to an agent to complete, and each task cannot be performed by more than one agent. 


\subsection{Multiple AUVS Collaborative Planning Assignments}

In multiple AUVs in many complex tasks require multiple AUV synergies to complete. Collaborative planning task allocation algorithm can undertake the task to complete decomposition and allocation for tasks require collaborative execution, the task can be broken down into several sub- tasks, and according to the agreement AUV developed jointly with other implementation plans [7-9].

Step one. The task commitment, Task description $\rightarrow A U V_{i}, A U V_{i}$ said underwater robots, Task description represents the task description.

Step two. Task decomposition, depending on the type and difficulty of the task undertaken by other factors, on the task decomposition, produce logic sub-tasks, sub-tasks execution order planning.

Step three. Mission planning, for each Task description, planning required number of underwater robots, can be expressed as a triple (AUV, Task description, Dependence), Dependence shows the relationship between underwater robots and tasks. $\left(A U V_{i}, T_{i}, \bigcup_{k=1}^{m}\left(A U V_{j k}, T_{j k}\right)\right)$ Expressed AUV $A U V_{i}$ Joint missions $T_{i}$, The task $T_{i}$ Decomposed into $m$ sub-tasks $T_{j k}$, By the appropriate underwater robot $A U V_{j k}$ Collaboration execution.

Step Fourpp. Assignments, using negotiation method for dynamic task allocation set D for negotiation domain; Negotiation is represented as a tuple.

Formula: Negotiation $=(A, R, \rho, N, U, P, S)$, in $A=\left\{A U V_{1}, \cdots, A U V_{k}\right\}(k \geq 2)$

underwater robot groups;

$R=\left\{r_{1}, \cdots, r_{k}\right\}(l \leq k)$ For the role sets;

$\rho$ for role assignment function, assign roles underwater robots,

$\rho(A U V)=r, A U V \in A, r \in R$;

$N \subseteq D$ For consultations set ;

$U=\left(u_{1}, \cdots u_{k}\right), u_{i}$ is $A U V_{i}$ The utility function ;

$P=\left(K,\left\{\pi: R \times K \rightarrow 2^{k} \mid r \in R\right\}\right)$ Expressed negotiated agreement ;

$S=\left\{\delta_{i}: P \times R \times K \times 2^{D} \times U \rightarrow K \times N \mid 1 \leq i \leq k\right\}$ Negotiation strategy represents a collection ;

Step Five. If the task allocation fails, go to step three;

Step Six. Mission planning completed.

\section{Experimental Results and Analysis}

\subsection{Multi-Tasking Comprehensive Superiority of AUVS}

Multiple AUVs common mobile search multiple dynamic targets, determine the angle of the advantages of multiple AUVs based on the initial velocity, angle advantage function is as follows.

$$
\mathrm{K}_{i j}^{a}=\frac{a_{j}-a_{i}}{180^{0}}
$$


S.t $\quad 0^{0} \leq a_{i}, a_{j} \leq 180^{\circ}$

$a_{i}$ Is the angle between the $i$ AUV search target when the target speed vector of the line of sight, $a_{j}$ Is the $j$ target and the angle between the $i$-th mobile agent and the target velocity vector of the line of sight.

According to multiple AUVs movement speed advantage, speed advantage function is as followspp.

$$
\mathrm{K}_{i j}^{v}=\left\{\begin{array}{l}
1, \quad v_{i}-v_{j}>0.1 \mathrm{~km} / \mathrm{s} \\
v_{i}-v_{j},\left|v_{i}-v_{j}\right| \leq 0.1 \mathrm{~km} / \mathrm{s} \\
-1, v_{i}-v_{j}<-0.1 \mathrm{~km} / \mathrm{s}
\end{array}\right.
$$

$v_{i}$ and $v_{j}$ The $\mathrm{i}$-th and $\mathrm{j}$-th AUV target velocity magnitude. When $\mathrm{K}_{i j}^{v}$ is positive , AUV speed represents the dominant, $\mathrm{K}_{i j}^{v}$ Is negative, indicating that the target movement speed predominate.

According to multiple AUVs moving distance advantage, distance advantage function is as followspp.

$$
\mathrm{K}_{i j}^{\mathrm{w}}=\left\{\begin{array}{l}
1, c_{i}-c_{j}>1 \mathrm{~km} \\
c_{i}-c_{j},\left|c_{i}-c_{j}\right| \leq 1 \mathrm{~km} \\
-1, c_{i}-c_{j}<-1 \mathrm{~km}
\end{array}\right.
$$

$c_{i}$ and $c_{j}$ The $\mathrm{i}$-th AUV can be moved a maximum distance, and the $\mathrm{j}$-th target can be moved a maximum distance .

Comprehensive advantages function is:

$k_{i j}=m_{a} k_{a}+m_{b} k_{v}+m_{c} k_{w}$

$k_{i j}$ Indicates the $\mathrm{i}$-th AUV Find comprehensive advantages of the $\mathrm{j}$-th target, $m_{a}$ 、 $m_{b}$ and $m_{c}$ Denote the angle advantage, speed advantage and the advantage of distance weighting factor, and $m_{a}+m_{b}+m_{c}=1$.

\subsection{Multi-Tasking AUVS Meta-Heuristic Ant Colony Algorithm}

Ant colony algorithm to calculate the meta-heuristic function $\eta_{i j}$,

$$
\eta_{i j}=\frac{1}{k_{i j}^{\prime}}
$$

$k_{i j}^{\prime}$ AUV represents a comparative advantage with dynamic target dummy value , $k_{i j}^{\prime}$ Smaller, the larger the $\eta_{i j}, p_{i j}^{k}$ greater. 


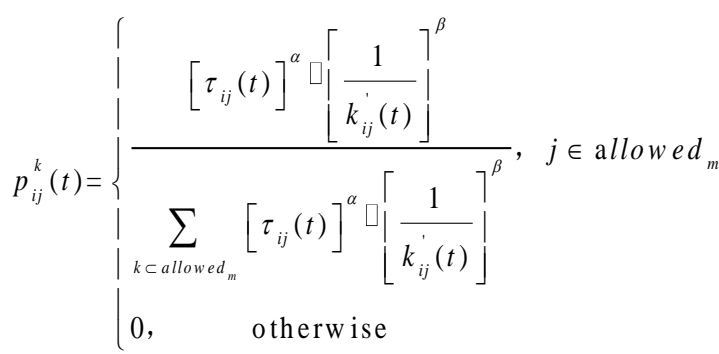

Consider $i$ AUV, then all the ants into $i$ groups, Consistent with the number of ants in each group is $n$, then the total number of ants target search of actual participation $i \times n$, each group of ants were searching for the $i$-th AUV comparative advantage biggest goal. In order to avoid repetition of ants searching for the same goal, therefore, Set $i$ taboo table, stored search targets have been identified, the ants cannot select the taboo target table already exists.

Taboo table is a $1 \times m_{l k}$ matrix, for searching for the number of dynamic targets. Taboo table stored only 0 and 1, If the $i$-th AUV has to find the first $j$-th target, $\mathrm{K}$-th goal in the taboo table position is set to 0, While the $j$-th goal in the $i$-th AUV taboo table, This is the element 0 of the search target had been selected as the taboo list. Thus, avoid AUV is always searching for the same goal, so you can successfully carry out the task assigned to all AUV, which can be solved multiple AUVs cooperative task allocation problem.

Ants search simultaneously on each AUV begins, each ant search after completion of the update again pheromone, according to the probability formula (3.6) to calculate the probability of selecting different search goals, When all the ants search is complete, select the biggest advantages of AUV search program, is about the strengths of each AUV search target value sum, Finally, the maximum value of the selected overall advantage to obtain the final result of multiple AUVs cooperative task assignment Multi AUVs cooperative task allocation algorithm .

\subsection{Simulation}

Initial parameters: $\alpha=1, \beta=3, \rho=0.6, \quad m=8, N_{C_{\max }}=150, Q=300$.Four AUV, Eight Is searching for the target, Each AUV assignments Up to two target Search.

Comparison of experimental results prove that the use of meta-heuristic ant colony optimization algorithm for solving multi- tasking aspect AUVs, solving performance than the basic ant colony algorithm has higher stability. 


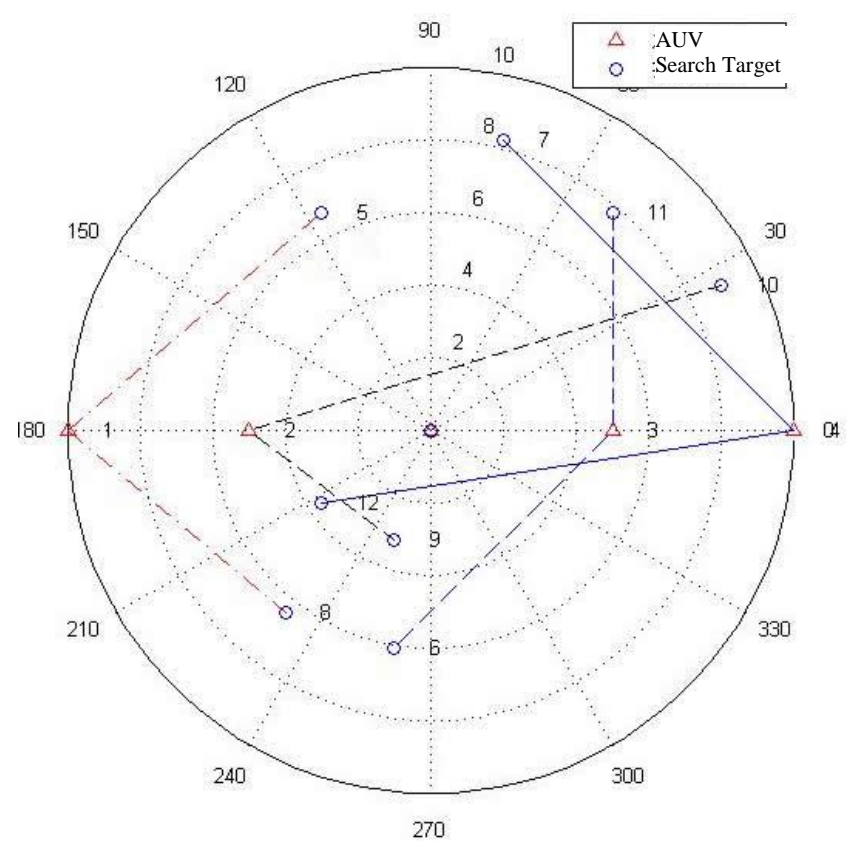

Figure 2. Search for Task Allocation Chart AUV

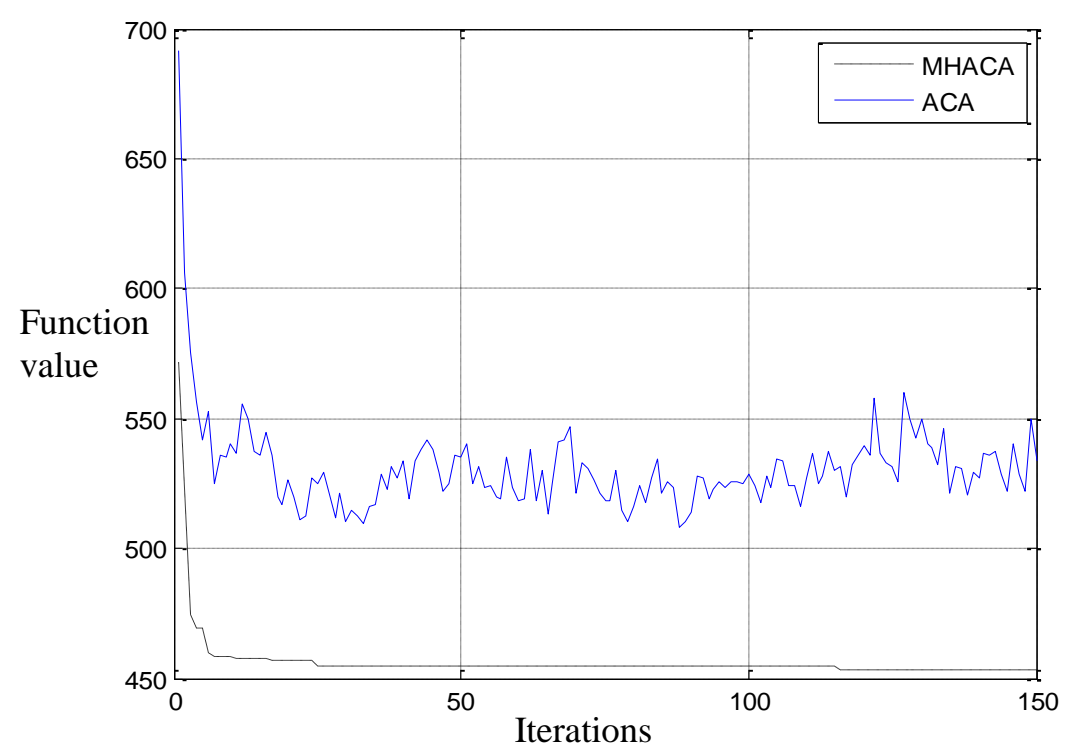

Figure 3. Meta- nt Heuristic AEvolution Curve Comparison

\section{Conclusions}

Thesis on meta-heuristic ant colony optimization algorithm and task allocation model was to describe the system, ant colony optimization algorithm is applied to multi- task allocation problem of underwater robots, through the comprehensive advantages of multitasking AUVs analysis, we propose a use ACO meta-heuristic methods to solve the multiAUVs cooperative task assignment, and has good scalability and application of simulation experiments to validate the method. 


\section{Acknowledgements}

This work was supported in part by the National Natural Science Foundation of China (60975071, 61100005), Ministry of Education, Scientific Research Project (13YJA790123), Heilongjiang Province Natural Science Fund Project (F201425).

\section{References}

[1] D. Q. Zhu, L. Xin and Y. M. Chong, "Multi- tasking multi autonomous underwater robot algorithms “, Control and Decision, vol. 8, (2012), pp. 89 -92.

[2] S. Fei, C. Yan and S. Lin-cheng, "Cooperative multitasking assignment based on ant colony algorithm for UAV “, Acta Aeronautica et Astronautica Sinica, vol. 5, (2008), pp. 184 -190.

[3] C. Jing, "PAN general", Improved ant colony algorithm for the same type of machine scheduling problem, Computer Engineering and Applications, vol. 47, (2011), pp. 44 -48.

[4] A. Colorni, M. Dorigo, V. Maniezzo, et. al., "Distributed optimization by ant colonies", Proceedings of the lst European Conference on Artificial Life, (1991), pp. 134-142, Paris.

[5] M. Dorigo, T. Stutzle, "Ant colony Optimization", MIT Press, (2004), Cambridge.

[6] K. Prescilla, "Real-time Task Assignment in Heterogeneous Multiprocessor Using Metaheuristic Algorithms", IEEE Xplore Advances in Computing and Communications (ICACC), 2013 Third International Conference on, vol. 8, (2013), pp. 248-252.

[7] Y. Deng, P. P. J. Beaujean, et. Al., "Task Allocation and Path Planning for Collaborative Autonomous Underwater Vehicles Operating through an Underwater Acoustic Network ", Hindawi Publishing Corporation Journal of Robotics Volume 2013, Article ID 483095, pp. 1-15.

[8] J. P. Wang, Y. Gu and X. M. LI, "Multi-robot Task Allocation Based on Ant Colony Algorithm", Journal Of Computers, vol. 7, no. 9, (2012), pp. 2160-2167.

[9] D. H. Bin , M. Jun , Z. Zhen, "fuzzy rule -based and dynamic ant - Assess the situation UCAV Bayesian network", CAAI Transactions on Intelligent Systems, vol. 04, (2013), pp. 119 -127.
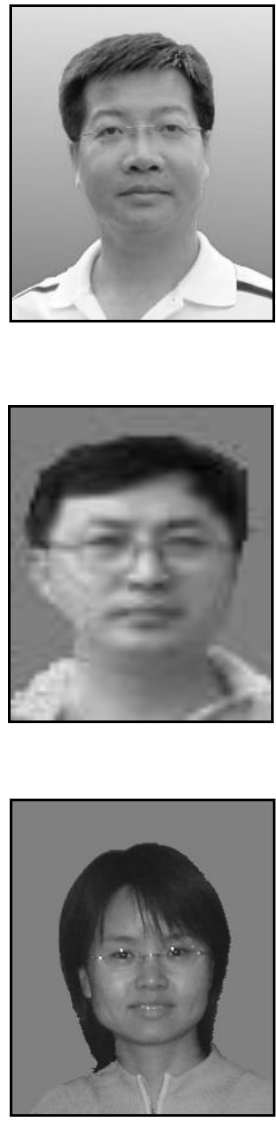

\section{Authors}

Jian Jun Li, he was born in 1973 year. He is a Ph.D. candidate at Harbin Engineering University. $\mathrm{He}$ is an Associate Professor at Harbin University of Commerce. His research interests include Business Intelligence, Machine Intelligence, etc.

RuBo Zhang, he was born in 1963 year. He is a professor and doctoral supervisor at Harbin Engineering University and Dalian Nationalities University. His research interests include Machine Intelligence, Artificial intelligence, etc.

Yu Yang, she was born in 1974 year. She is an Associate Professor at Harbin University of Commerce. Her research interests include E-commerce, Business Intelligence, etc. 
International Journal of Grid Distribution Computing Vol. 8, No. 3, (2015) 